\title{
Analysis of Spontaneous EEG Activity in Alzheimer's Disease Using Cross-Sample Entropy and Graph Theory
}

\author{
Carlos Gómez, IEEE Senior Member, Jesús Poza, IEEE Senior Member, Javier Gomez-Pilar, IEEE \\ Student Member, Alejandro Bachiller, IEEE Student Member, Celia Juan-Cruz, \\ Miguel A. Tola-Arribas, Alicia Carreres, Mónica Cano, and Roberto Hornero, IEEE Senior Member
}

\begin{abstract}
The aim of this pilot study was to analyze spontaneous electroencephalography (EEG) activity in Alzheimer's disease (AD) by means of Cross-Sample Entropy (Cross-SampEn) and two local measures derived from graph theory: clustering coefficient $(C C)$ and characteristic path length $(P L)$. Five minutes of EEG activity were recorded from 37 patients with dementia due to $A D$ and 29 elderly controls. Our results showed that Cross-SampEn values were lower in the AD group than in the control one for all the interactions among EEG channels. This finding indicates that EEG activity in AD is characterized by a lower statistical dissimilarity among channels. Significant differences were found mainly for fronto-central interactions $(p<0.01$, permutation test). Additionally, the application of graph theory measures revealed diverse neural network changes, i.e. lower $C C$ and higher $P L$ values in AD group, leading to a less efficient brain organization. This study suggests the usefulness of our approach to provide further insights into the underlying brain dynamics associated with AD.
\end{abstract}

\section{INTRODUCTION}

Alzheimer's disease (AD) is a primary degenerative dementia, which gradually destroys brain cells. The number of people with dementia increases exponentially with increasing age. $\mathrm{AD}$ affects $1 \%$ of population under 60 years but reaches $30 \%$ for people over 85 years. Besides, due to the increase in average life expectancy during the 21 st century, it is expected that dementia will affect 81.1 million people by 2040 [1]. Thus, AD is becoming a key public-healthcare and economic problem.

Pathologically, AD is characterized by the deposition of amyloid beta-peptide as insoluble extracellular plaques and intracellular tau aggregation [2]. Currently, a definite diagnosis of $\mathrm{AD}$ requires examination of brain tissue at autopsy. For this reason, physicians can really only make a diagnosis of probable AD. Clinical diagnosis usually includes medical history, neurological examination, blood tests, and neuroimaging studies. Nowadays, electroencephalography

This research was supported in part by 'Ministerio de Economía y Competitividad' and FEDER under project TEC2014-53196-R, 'Consejería de Educación de la Junta de Castilla y León' under project VA059U13, and 'Consejería de Sanidad de la Junta de Castilla y León' under project $\mathrm{BIO} / \mathrm{VA} 08 / 15$

C. Gómez, J. Poza, J. Gomez-Pilar, A. Bachiller, C. Juan-Cruz, and R. Hornero are with the Biomedical Engineering Group, E.T.S. Ingenieros de Telecomunicación, University of Valladolid, Paseo Belén 15, 47011 Valladolid, Spain (e-mail: carlos.gomez@tel.uva.es).

M. A. Tola and A. Carreres are with the Department of Neurology, Hospital Universitario Río Hortega, Valladolid, Spain.

M. Cano is with the Department of Clinical Neurophysiology, Hospital Universitario Río Hortega, Valladolid, Spain.
(EEG) is not always used in AD diagnosis, in spite of its potential to characterize neural dynamics [3]. EEG is a noninvasive technique to measure the electromagnetic brain activity [4]. It allows the assessment of the spatio-temporal patterns of neural activity and their interactions in the time range of milliseconds [5]. The EEG reflects not only brain oscillation activity but also the underlying associated neural dynamics [6]. As AD affects the cerebral cortex areas, EEG is a useful technique to understand the neural mechanisms of this disorder.

For the analysis of EEG activity in $\mathrm{AD}$, spectral techniques are the most extended option, due to their simplicity and ease of clinical interpretation. Spectral analyses seem to discriminate $\mathrm{AD}$ patients from control subjects through an increased brain activity in lower frequency bands associated with AD [7]. From another point of view, non-linear analysis techniques have been widely used since the mid-90s [8]. Several non-linear analysis methods have suggested that AD elicits a complexity decrease in spontaneous brain activity (using Lempel-Ziv complexity or fractal dimension) and an increase of regularity (using approximate entropy and sample entropy) [9-10]. All these methods (both spectral and non-linear) measure local activation patterns in individual sensors. However, it has become clear that simple activation studies are no longer sufficient for AD characterization [11]. For this reason, graph theory has been introduced in cognitive neuroscience to overcome the limitations of the aforementioned analyses. In this regard, the application of graph theory concepts can be used to gain more insight into the $\mathrm{AD}$ pathophysiological processes [12].

In this study, EEG activity has been analyzed in 37 patients with dementia due to $\mathrm{AD}$ and 29 control subjects using Cross-Sample Entropy (Cross-SampEn) and two local measures derived from graph theory: clustering coefficient $(C C)$ and characteristic path length $(P L)$. Our aim is to characterize the neural mechanisms in AD.

\section{MATERIALS}

\section{A. Subjects}

EEG data were acquired from 66 subjects. The AD group was formed by thirty-seven patients with mild/moderate dementia due to $\mathrm{AD}$, diagnosed according to the clinical criteria of the National Institute on Aging and Alzheimer's Association (NIA-AA). Twenty-nine elderly subjects without cognitive impairments and with no history of neurological or psychiatric disorders comprised the control group. Table 1 shows socio-demographic and clinical data for both groups. 
TABLE I. SOCIO-DEMOGRAPHIC AND CLINICAL DATA FOR EACH GROUP

\begin{tabular}{|c|c|c|}
\cline { 2 - 3 } \multicolumn{1}{c|}{} & AD group & Control group \\
\hline Number of subjects & 37 & 29 \\
\hline Age (years) (Mean \pm SD) & $79.2 \pm 6.9$ & $76.6 \pm 7.2$ \\
\hline Gender (Male:Female) & $13: 24$ & $9: 20$ \\
\hline MMSE $^{\text {a }}$ (Mean \pm SD) & $18.7 \pm 6.2$ & $28.9 \pm 1.3$ \\
\hline
\end{tabular}

All participants and patients' caregivers were informed about the research background and the study protocol. Moreover, all of them gave their written informed consent to be included in the study. The Ethics Committee at the "Hospital Universitario Río Hortega" (Valladolid, Spain) endorsed the study protocol, according to The Code of Ethics of the World Medical Association (Declaration of Helsinki).

\section{B. EEG recording}

EEG activity was acquired with a 19-channel EEG system $\left(\mathrm{XLTEK}^{\circledR}\right.$, Natus Medical). For each subject, five minutes of resting-state EEG activity were recorded at a sampling frequency of $200 \mathrm{~Hz}$ from the 19 following electrodes: Fp1, Fp2, Fz, F3, F4, F7, F8, Cz, C3, C4, T3, T4, T5, T6, Pz, P3, P4, O1 and O2. EEG data were then digitally filtered between 1 and $40 \mathrm{~Hz}$. Finally, $27.7 \pm 8.4$ (mean \pm standard deviation, SD) artifact-free 5-s length epochs per channel and subject were selected by visual inspection.

\section{METHODS}

\section{A. Cross Sample Entropy (Cross-SampEn)}

Cross-SampEn is a nonlinear measure that allows assessing the degree of similarity of two time series [13]. It was proposed by Richman and Moorman to overcome some drawbacks of a related algorithm called Cross-Approximate Entropy (Cross-ApEn). Firstly, Cross-SampEn remains relatively consistent for conditions where Cross-ApEn does not. Secondly, Cross-ApEn is not always defined. CrossSampEn solves this problem. Finally, there is other important difference between these two algorithms: whereas Cross$A p E n$ analysis exhibits direction dependence (i.e. it is an asymmetric method), Cross-SampEn is a direction independent measure.

To compute Cross-SampEn, a run length $m$ and a tolerance window $r$ must been set. Given two time series, $u(i)$ and $v(i)$, Cross-SampEn measures, within tolerance $r$, the frequency of $v$-patterns similar to a given $u$-pattern of length $m$. Although a right choice of $m$ and $r$ are important to calculate Cross-SampEn, no guidelines exist to optimize their values. However, values of $m$ equal to 1 or 2 , and $r$ between 0.1 and 0.25 have been suggested [14]. In this pilot study, we have specified values of $m=1$ and $r=0.2$. The detailed algorithm to calculate the Cross-SampEn between two signals $u(i)$ and $v(i)$ is the following $[13,15]$ :

1) Normalize $u(i)$ and $v(i)$. The normalized time series $u^{*}(i)$ and $v^{*}(i)$ are:

$$
\begin{aligned}
& u^{*}(i)=[u(i)-\operatorname{mean}(u)] / \mathrm{SD}(u) \\
& v^{*}(i)=[v(i)-\operatorname{mean}(v)] / \mathrm{SD}(v) .
\end{aligned}
$$

2) Form the vector sequences:

$$
\begin{array}{ll}
x_{m}(i)=\left[u^{*}(i), u^{*}(i+1), \ldots, u^{*}(i+m-1)\right], & 1 \leq i \leq N-m \\
y_{m}(j)=\left[v^{*}(j), v^{*}(j+1), \ldots, v^{*}(j+m-1)\right], & 1 \leq j \leq N-m .
\end{array}
$$

3) Define the distance between the vector sequences $x_{m}(i)$ and $y_{m}(j)$ as:

$$
d\left[x_{m}(i), y_{m}(j)\right]=\max _{k=0,1, \ldots, m-1}\left|u^{*}(i+k)-v^{*}(j+k)\right| .
$$

4) For each $x_{m}(i)$, count the number of $j(j=1,2, \ldots, N-m)$ so that $d\left[x_{m}(i), y_{m}(j)\right] \leq r$, denoted as $b_{i}^{m}(r)$. Then, for $i=1,2, \ldots, N-m$, set:

$$
B_{i}^{m}(r)(v \| u)=\frac{b_{i}^{m}(r)}{N-m} .
$$

$B_{i}^{m}(r)(v \| u)$ is the probability that any $y_{m}(j)$ is within $r$ of $x_{m}(i)$.

5) Define $B^{m}(r)(v \| u)$ as:

$$
B^{m}(r)(v \| u)=\frac{1}{N-m} \sum_{i=1}^{N-m} B_{i}^{m}(r)(v \| u) .
$$

6) Similarly, define $A_{i}^{m}(r)(v \| u)$ as $1 /(N-m)$ times the number of $j(j=1,2, \ldots, N-m)$, such the distance between $x_{m+1}(i)$ and $y_{m+1}(j)$ is less than or equal to $r$. Then, calculate:

$$
A^{m}(r)(v \| u)=\frac{1}{N-m} \sum_{i=1}^{N-m} A_{i}^{m}(r)(v \| u) .
$$

$B^{m}(r)(v \| u)$ is the probability that two templates matches for $m$ points, whereas $A^{m}(r)(v \| u)$ is the probability that two templates matches for $m+1$ points.

7) Finally, Cross-SampEn is defined as:

$$
\text { Cross - SampEn }(m, r, N)(v \| u)=-\ln \left[\frac{A^{m}(r)(v \| u)}{B^{m}(r)(v \| u)}\right] \text {. }
$$

In this study, Cross-SampEn has been computed for all pairwise combinations of EEG channels. After the averaging process over all artifact-free epochs, a $19 \times 19$ Cross-SampEn matrix per subject was obtained.

\section{B. Graph Theory}

Graph theory is the scientific study of graphs or networks. A graph is a set of nodes (or vertices) and the corresponding set of edges (or connections), defined as the connectivity/similarity values among nodes [16]. In our study, a network with $N=19$ nodes (corresponding to the aforementioned 19 EEG electrodes) was analyzed. The edge between two vertices $k$ and $l$ takes the Cross-SampEn value between channels $k$ and $l$. The edge weight between these two vertices $k$ and $l$ is denoted as $w_{k l}$. A graph can be characterized using various network parameters. In this study, $C C$ and $P L$ were calculated for each node.

We can define the $C C$ of a node $k, C C_{k}$, as the probability that other nodes $l$, that are connected to node $k$, will be also connected to each other [17]: 


$$
C C_{k}=\frac{\sum_{p \neq k} \sum_{\substack{q \neq k \\ q \neq p}} w_{k p} w_{k q} w_{p q}}{\sum_{\substack{p \neq k \\ q \neq k \\ q \neq p}} \sum_{k p} w_{k q}} .
$$

On the other hand, $P L$ of node $k, P L_{k}$, is calculated as the average minimal travel distance $d_{k l}$ between node $k$ and all nodes $l[18]$ :

$$
P L_{k}=\frac{\sum_{l \neq k} d_{k l}}{N-1},
$$

where $d_{k l}$ is the shortest weighted distance between nodes $k$ and $l$ in the graph and $N$ is the number of nodes of the network $(N=19)$.

Control group
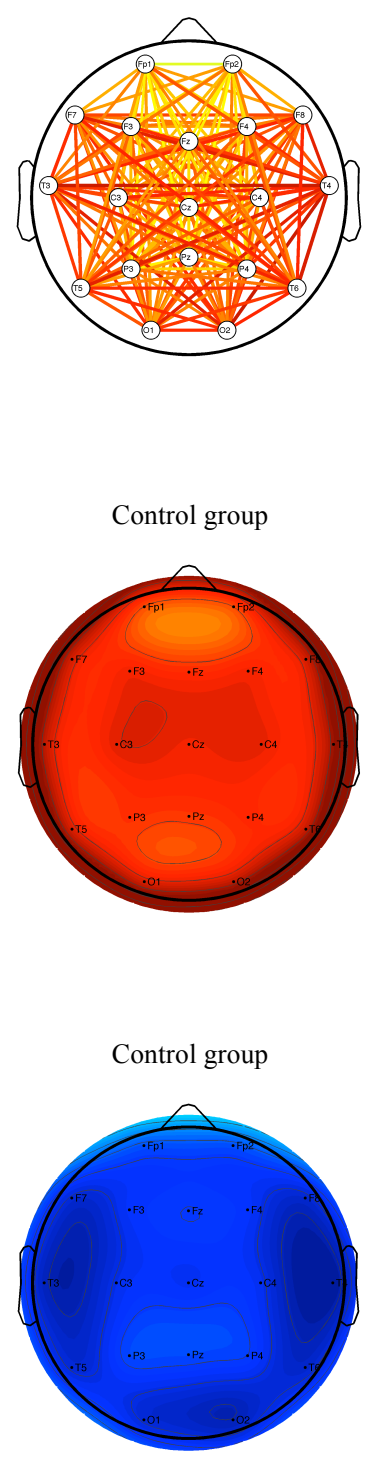

AD group

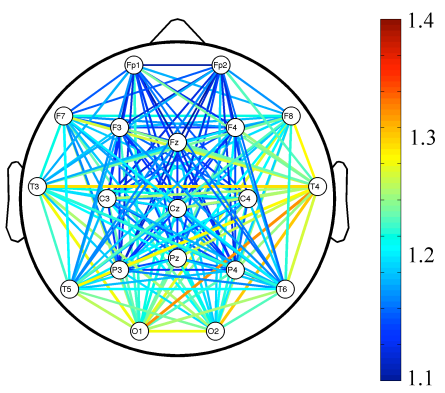

(a)

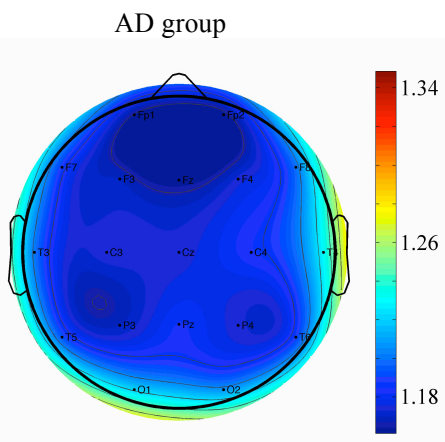

(b)

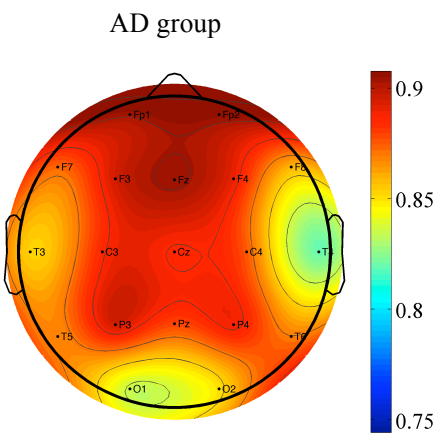

(a)

\section{Statistical analysis}

Statistical comparison between groups was performed by means of multiple comparisons nonparametric permutation test. It allows achieving a strong control over type I error in situations in which the multiplicity of testing must be taken into account, as in this case.

\section{RESUlTS}

Firstly, Cross-SampEn measure was applied to the EEG data with parameter values of $m=1$ and $r=0.2$. Fig. 1a illustrates entropy values for both groups. This figure shows that Cross-SampEn values were higher in the control group than in AD group for all EEG channels combinations. This finding suggests that dementia due to AD is characterized by a higher degree of similarity among channels. Right panel of Fig. 1a summarized statistical results (it is important to note
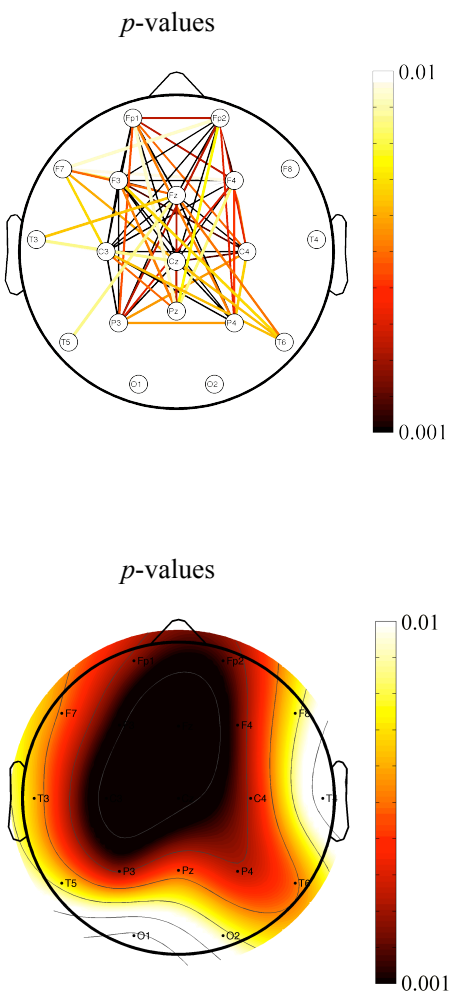

(c)

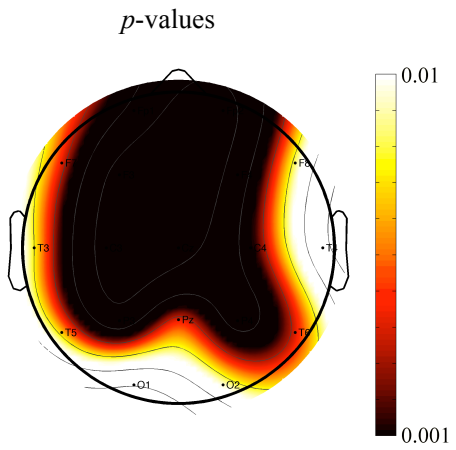

Figure 1. EEG layouts showing the distribution of mean values for each group (Controls and AD patients) and the corresponding $p$-values: (a) Cross-Sample Entropy among electrodes; (b) Clustering coefficient of each electrode; (c) Characteristic path length of each electrode. 
that only significant differences between groups are displayed).

Secondly, $C C$ and $P L$ measures were calculated from Cross-SampEn values. As expected, our results revealed that $\mathrm{AD}$ is associated with lower $C C$ in comparison with controls. On the other hand, the patients group exhibited higher values of $P L$ than the control group. Differences were statistically significant ( $p<0.01$, nonparametric permutation test) with both parameters in all EEG channels, with the exception of $\mathrm{T} 4$ and $\mathrm{O} 1$.

\section{DiscuSSION AND CONCLUSION}

In this pilot study, EEG activity was analyzed from 37 patients with dementia due to $\mathrm{AD}$ and 29 elderly control subjects by means of Cross-SampEn, CC and PL. Our main goal was to characterize the neural mechanisms in AD.

Cross-SampEn results revealed that AD patients exhibit a higher degree of similarity among channels than controls. These findings agree with previous studies. For instance, Jeong et al. [19] reported lower values of cross-mutual information in $\mathrm{AD}$ subjects than in controls, mainly over frontal and antero-temporal regions. Koenig et al. [20] analyzed a large EEG database of control subjects and patients with varying degrees of cognitive decline or AD using global field synchronization. Their results showed decreased synchronization values in alpha, beta, and gamma frequency bands [20]. All these studies support the notion that $\mathrm{AD}$ is associated with a loss of functional connectivity.

Graph theory results showed lower $C C$ and higher $P L$ values in $\mathrm{AD}$ group in comparison with the control one. As $C C$ provides a measure of the node strength, our study indicates that functional brain networks in $\mathrm{AD}$ are characterized by a diminished strength. On the other hand, our $P L$ results suggest that the different brain areas are worse connected in AD group. These findings partially agree with previous studies. Using synchronization likelihood and unweighted graphs, de Haan et al. [12] found a significant decrease of $C C$ in the lower alpha and beta bands in AD group, but also diminished $P L$ values in the lower alpha and gamma bands. The main finding of an fMRI study is that brain networks in $\mathrm{AD}$ are characterized by lower $C C$ values but similar $P L$ values in comparison with controls [21]. These researches suggest that $\mathrm{AD}$ is associated with less efficient information exchange between brain areas [12].

Some limitations of our research work merit consideration. Firstly, the sample size was limited to 66 subjects. In addition to this, only AD patients and controls took part in this study. Future efforts will be focused to increase the number of subjects, as well as to include mild cognitive impairment subjects, as this condition is considered a prodromal stage of AD. Finally, $C C$ and $P L$ are not the only measures to characterize brain networks, so other graph theory parameters could provide complementary information.

In sum, our study leads us to conclude that spontaneous EEG activity in AD patients is accompanied by diverse neural network changes. This study suggests the usefulness of our approach (combination of Cross-SampEn, CC and PL) to provide further insights into the underlying brain dynamics associated with AD.

\section{REFERENCES}

[1] C. P.Ferri, M. Prince, C. Brayne, H. Brodaty, L. Fratiglioni, M. Ganguli, et al., "Global prevalence of dementia: a Delphi consensus study," Lancet, vol. 366, pp. 2112-2117, 2006.

[2] B. P.Lucey and D. M. Holtzman, "How amyloid, sleep and memory connect," Nature Neurosci., vol. 18, pp. 933-934, 2015.

[3] J. Jeong, "EEG dynamics in patients with Alzheimer's disease," Clin. Neurophysiol., vol. 115, pp. 1490-1505, 2004.

[4] B. J. Roach and D. H. Mathalon, "Event-related EEG time-frequency analysis: an overview of measures and an analysis of early gamma band phase locking in schizophrenia," Schizophr. Bull., vol. 34, pp. 907-926, 2008.

[5] T. P. Meehan and S. L. Bressler, "Neurocognitive networks: Findings, models, and theory," Neurosci. Biobehav. R., vol. 36, pp. 2232-2247, 2012.

[6] O. A. Rosso, M. T. Martin, A. Figliola, K. Keller, and A. Plastino, "EEG analysis using wavelet-based information tools," J. Neurosci. Methods, vol. 153, pp. 163-182, 2006.

[7] M. Signorino, E. Pucci, N. Belardinelli, G. Nolfe, and F. Angeleri, "EEG spectral analysis in vascular and Alzheimer dementia," Electroencephalogr. Clin. Neurophysiol., vol. 94, pp. 313-325, 1995.

[8] C. J. Stam, "Nonlinear dynamical analysis of EEG and MEG: review of an emerging field," Clin. Neurophysiol., vol. 116, pp. 2266-2301, 2005.

[9] C. Gómez, R. Hornero, D. Abásolo, A. Fernández, and J. Escudero, "Analysis of MEG background activity in Alzheimer's disease using non-linear methods and ANFIS," Ann. Biomed. Eng., vol. 37, pp. 586594, 2009.

[10] C. Gómez, A. Mediavilla, R. Hornero, D. Abásolo, and A. Fernández, "Use of the Higuchi's fractal dimension for the analysis of MEG recordings from Alzheimer's disease patients," Med. Eng. Phys., vol. 31, pp. 306-313, 2009.

[11] C. J. Stam and E. C. W. van Straaten, "The organization of physiological brain networks," Clin. Neurophysiol., vol. 123, pp. 1067-1087, 2012.

[12] W. de Haan, Y. A. Pijnenburg, R. L. Strijers, Y. van der Made, W. M. van der Flier, P. Scheltens, and C. J. Stam, "Functional neural network analysis in frontotemporal dementia and Alzheimer's disease using EEG and graph theory," BMC Neurosci., vol. 10, pp. 101, 2009.

[13] J. S. Richman and J. R. Moorman, "Physiological time-series analysis using approximate entropy and sample entropy," Am. J. Physiol. Heart Circ. Physiol., vol. 278, pp. H2039-H2049, 2000.

[14] S. M. Pincus, "Irregularity and asynchrony in biologic network signals," Methods Enzymol., vol. 321, pp. 149-182, 2000.

[15] W. Shi and P. Shang, "Cross-sample entropy statistic as a measure of synchronism and cross-correlation of stock markets," Nonlinear Dynam., vol. 71, pp. 539-554, 2013.

[16] S. C. Ponten, A. Daffertshofer, A. Hillebrand, and C. J. Stam, "The relationship between structural and functional connectivity: graph theoretical analysis of an EEG neural mass model," Neuroimage, vol. 52, pp. 985-994, 2010.

[17] C. J. Stam, W. de Haan, A. Daffertshofer, B. F. Jones, I. Manshanden, A. M. van Cappellen van Walsum, et al., "Graph theoretical analysis of magnetoencephalographic functional connectivity in Alzheimer's disease," Brain, vol. 132, pp. 213-224, 2009.

[18] M. P. van den Heuvel, R. C. W. Mandl, C. J. Stam, R. S. Kahn, and H. E. H. Pol, "Aberrant frontal and temporal complex network structure in schizophrenia: a graph theoretical analysis," J. Neurosci., vol. 30, pp. 15915-15926, 2010.

[19] J. Jeong, J. C. Gore, and B. S. Peterson, "Mutual information analysis of the EEG in patients with Alzheimer's disease," Clin. Neurophysiol., vol. 112, pp. 827-835, 2001.

[20] T. Koenig, L. Prichep, T. Dierks, D. Hubl, L. O. Wahlund, E. R. John, and V. Jelic, "Decreased EEG synchronization in Alzheimer's disease and mild cognitive impairment," Neurobiol. Aging, vol. 26, pp. 165$171,2005$.

[21] K. Supekar, V. Menon, D. Rubin, M. Musen, and M. D. Greicius, "Network analysis of intrinsic functional brain connectivity in Alzheimer's disease," PLoS Comput. Biol., vol. 4, pp. e1000100, 2008. 